\title{
Dye-Free Porcine Model of Experimental Branch Retinal Vein Occlusion: A Suitable Approach for Retinal Proteomics
}

\author{
Lasse Jørgensen Cehofski, ${ }^{1,2}$ Anders Kruse, ${ }^{1}$ Benedict Kjærgaard, ${ }^{2,3}$ Allan Stensballe, ${ }^{4}$ \\ Bent Honoré, ${ }^{5}$ and Henrik Vorum ${ }^{1}$ \\ ${ }^{1}$ Department of Ophthalmology, Aalborg University Hospital, Hobrovej 18-22, 9000 Aalborg, Denmark \\ ${ }^{2}$ Biomedical Research Laboratory, Aalborg University Hospital, Ladegårdsgade 3, 9000 Aalborg, Denmark \\ ${ }^{3}$ Department of Heart and Lung Surgery, Center for Cardiovascular Research, Aalborg University Hospital, Hobrovej 18-22, \\ 9000 Aalborg, Denmark \\ ${ }^{4}$ Department of Health Science and Technology, Aalborg University, Fredrik Bajers Vej 3, Building B, 9220 Aalborg, Denmark \\ ${ }^{5}$ Department of Biomedicine, Aarhus University, Ole Worms Allé 3, Building 1182, Room 024, 8000 Aarhus C, Denmark
}

Correspondence should be addressed to Henrik Vorum; henrik.vorum@rn.dk

Received 15 January 2015; Accepted 29 March 2015

Academic Editor: Theodore Leng

Copyright (C) 2015 Lasse Jørgensen Cehofski et al. This is an open access article distributed under the Creative Commons Attribution License, which permits unrestricted use, distribution, and reproduction in any medium, provided the original work is properly cited.

\begin{abstract}
Branch retinal vein occlusion induces complex biological processes in the retina that are generated by a multitude of interacting proteins. These proteins and their posttranslational modifications can effectively be studied using modern proteomic techniques. However, no method for studying large-scale protein changes following branch retinal vein occlusion has been available until now. Obtainment of retinal tissue exposed to branch retinal vein occlusion is only available through experimental animal models. Traditional models of experimental branch retinal vein occlusion require the use of Rose Bengal dye combined with argon laser photocoagulation. The use of Rose Bengal dye is problematic in proteomic studies as the dye can induce multiple protein modifications when irradiated. This paper presents a novel technique for proteomic analysis of porcine retinal tissue with branch retinal vein occlusion combining a dye-free experimental model with label-free liquid chromatography mass spectrometry based proteomics.
\end{abstract}

\section{Introduction}

Branch retinal vein occlusion (BRVO) is the second most frequent retinal vascular disease after diabetic retinopathy [1]. The disease is a common cause of visual loss in middle aged and elderly people [2]. The predominant pathogenetic mechanism for BRVO is thought to be compression of the retinal vein by a thickened retinal artery [2] that occurs at a site of arteriovenous crossing [3]. This mechanical narrowing of the venous lumen results in widespread flame-shaped haemorrhages, axonal congestion, dilated tortuous veins, ischaemia, and oedema upstream of the occlusion $[2,4]$.

In retinal tissue BRVO induces complex biological processes that are driven by a multitude of interacting proteins.
For example, the VEGF-VEGF2 signalling pathway has been shown to have an essential role in BRVO [5]. Large-scale protein changes following BRVO can effectively be studied using proteomic technologies to bring new insights into pathological processes and signaling pathway alterations that occur in the disease [6-8]. However, no method for analyzing large-scale protein changes following BRVO has been established.

From human eyes with BRVO, retinal tissue samples are generally only obtainable at postmortem $[9,10]$ which makes experimental models of large animals better suited for studying retinal protein changes using proteomics [11]. Porcine retinas are highly suitable for experimental studies of BRVO. Like the human retina the porcine retina is fully 
vascularised which is an advantage if hypoperfusion and ischemia develop [12]. Furthermore, porcine and human eyes are largely identical in size [12]. Therefore, porcine eyes may render a sufficient amount of retinal tissue for proteomic studies. In addition, the size of porcine eyes makes them suitable for validation with fluorescein angiography and fundus photography [13].

Existing experimental models of BRVO are predominantly based on photothrombosis [14-16]. With photothrombosis the animal receives an intravenous injection of Rose Bengal dye followed by application of argon green laser burns directly on the branch vein which creates an intravascular thrombus $[14,15]$. The peak absorption of Rose Bengal is close to the wavelength of argon laser which makes it possible to use less laser energy [17].

When exposed to visible light Rose Bengal can induce posttranslational modifications. Therefore, experimental BRVO is best induced without the use of Rose Bengal if the retinal tissue is intended for proteomic experiments. In this paper we introduce a novel method that allows for largescale protein identification of more than 2000 proteins in porcine retinal tissue exposed to experimental BRVO by combining a dye-free experimental model with label-free liquid chromatography mass spectrometry based proteomics.

\section{Materials and Methods}

The study was approved by the Danish Animal Experiments Inspectorate (permission number 2013-15-2934-00775). A Danish Landrace pig, approximately $30 \mathrm{~kg}$, was anesthetized with an intramuscular injection of Zoletil (ketamine $6.25 \mathrm{mg} /$ $\mathrm{mL}$, tiletamine $6.25 \mathrm{mg} / \mathrm{mL}$, zolazepam $6.25 \mathrm{mg} / \mathrm{mL}$, butorphanol $1.25 \mathrm{mg} / \mathrm{mL}$, and xylain $6.25 / \mathrm{mL}$ ).

2.1. Experimental Branch Retinal Vein Occlusion. The eyes were anesthetized with Oxybuprocaine Hydro 0.4\% (Bausch \& Lomb) and Tetracaine 1\% (Bausch \& Lomb) followed by dilatation with Tropicamide 0.5\% (Mydriacyl; Bausch \& Lomb) and Phenylephrine 10\% (Metaoxidrin; Bausch \& Lomb). To prevent the corneal surface from becoming dry and compromise the view of the retina Systane Ultra eye drops (Polyethylene Glycol 400, Propylene Glycol; Alcon, Copenhagen, Denmark) were applied every 5 minutes.

BRVO was induced using a standard argon laser $(532 \mathrm{~nm})$ that was given by indirect ophthalmoscopy. Initially laser burns were applied around an inferior branch vein to narrow the vessel. The following laser application was given directly on the vein until stagnation of the venous blood flow occurred. After 37 laser applications with a power of $400 \mathrm{~mW}$ and a duration of $550 \mathrm{~ms}$ the venous blood stagnated in the vein (Figure 1). Peripheral flame-shaped hemorrhages appeared within 30 minutes after the occlusion had been induced.

In the inferior retina of the left eye that served as a control, laser was applied in a similar area that was free of vessels. A validating fluorescein angiography was conducted nine days after induction of the BRVO to confirm

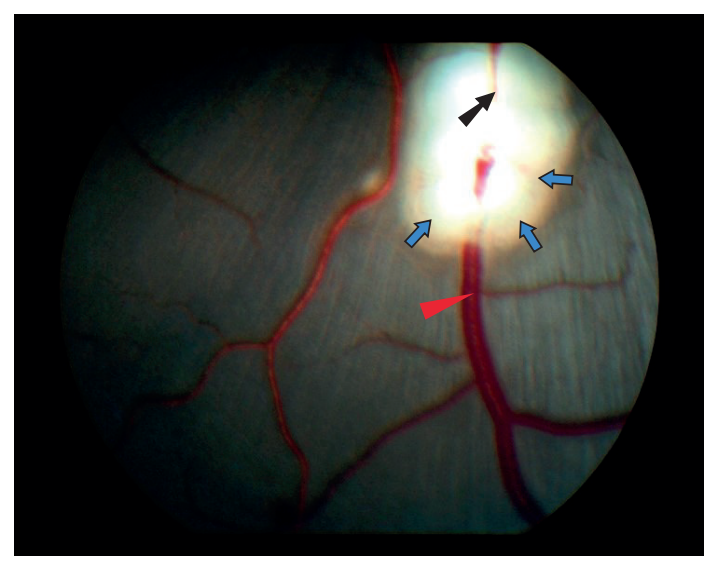

FIGURE 1: Dilation of vein due to downstream occlusion. The dilated section of the vein is marked with red triangle. The laser patch is marked with blue arrow. The site of occlusion is marked with black arrow.

successful occlusion. By day 15 the pig was examined with ophthalmoscopic examination and fundus photography followed by enucleation of the eyes that were stored at $-80^{\circ} \mathrm{C}$ until preparation for proteomic analysis. Euthanasia of the pig followed immediately after enucleation. The right eye with $\mathrm{BRVO}$ was used for proteomic analysis.

2.2. Sample Preparation for Proteomic Analysis. The porcine eye was thawed on ice. A circumferential incision was made around the iris $2 \mathrm{~mm}$ posteriorly to the limbus to remove the cornea and the iris. The lens and the vitreous body were carefully lifted and the retina was peeled from the eye cup and placed in an Eppendorf tube with five $2.3 \mathrm{~mm}$ chromium balls. The Eppendorf tube was placed on a Vortex mixer for approximately 30 seconds to homogenize the sample. A volume of $500 \mu \mathrm{L}$ cold PBS was added to the Eppendorf tube followed by another 30 seconds of homogenization on the Vortex mixer. A volume of $50 \mu \mathrm{L}$ of the homogenate was placed in a bead beading tube containing $500 \mu \mathrm{L} \mathrm{5 \%}$ sodium deoxycholate ( $\mathrm{NaDOC}), \mathrm{pH} 8.5$, and homogenized by bead beading with a Precellys 24 homogenizer (Bertin Technologies, Rockville, MD, USA) at 6,000 rpm for $20 \mathrm{sec}$ per round. The samples were kept on ice between bead beading runs. The retinal sample was placed in a YM10 Spin Filter (Millipore) and buffer exchange to digestion buffer (1\% NaDOC in 0.1 M TEAB; pH 7.8) was performed. This was followed by reduction at $37^{\circ} \mathrm{C}$ using $12 \mathrm{mM}$ TCEP for 30 minutes and alkylation with $40 \mathrm{mM}$ iodoacetamide for 30 minutes followed by two times buffer exchange by centrifugation at $14.400 \mathrm{~g}$ into $0.5 \% \mathrm{NaDOC}$ in $0.1 \mathrm{M} \mathrm{TEAB}$; $\mathrm{pH}$ 7.8. For digestion two ug trypsin (modified, Promega) was added for incubation overnight. The filtrate was recovered and sodium deoxycholate was precipitated by adding $5 \%$ formic acid followed by recovery of the soluble peptides. An internal standard of $20 \mathrm{fm} / \mathrm{uL}$ BSA digest (Waters Massprep) was added and the retinal sample was analyzed by nanoUPLC MS. 
2.3. Liquid Chromatography Tandem Mass Spectrometry Data Acquisition. A sample volume of $5 \mu \mathrm{L}$ was transferred onto a Dionex RSLC nano-UPLC system connected to a Quadrupole Orbitrap (Q Exactive Plus) mass spectrometer that was equipped with a NanoSpray Flex ion source (Thermo Scientific, Bremen, Germany). The sample was loaded onto a trapping column (Acclaim PepMap100 C18, $5 \mu \mathrm{m}$ column from Thermo Scientific) at a flow rate of $8 \mu \mathrm{L}$ per min. Peptide separation was conducted at a nanoflow of $300 \mathrm{~nL}$ per min on the analytical column $(50 \mathrm{~cm}$ Acclaim PepMap RSLC). The nanoelectrospray was conducted using a Picotip "Silicatip" emitter from New Objectives. Formic acid 0.1\% (buffer A) and $99.9 \%$ acetonitrile with formic acid $0.1 \%$ (buffer B) were used as buffers. The applied gradient ranged between $10 \%$ and $30 \%$ over 230 minutes.

Operating in data-dependent acquisition mode a full MS scan in the mass range of 350 to $1400 \mathrm{~m} / z$ was obtained at a resolution of 70,000 with an AGC target of $10^{6}$ and maximum fill time set to $100 \mathrm{~ms}$. The contaminant ion at 391.28429 $\mathrm{m} / \mathrm{z}$ was used for instrument lock mass correction. Up to $12 \mathrm{MS} / \mathrm{MS}$ acquisitions on abundant peptide precursor ions were triggered per cycle. The MS/MS scans were acquired with a dynamic mass range at a resolution of 17,500 and with an AGC target of $5 \times 10^{5}$ and max fill time of $50 \mathrm{~ms}$. A quadrupole isolation window of $2.0 \mathrm{~m} / z$ was used for isolation of the precursor ions that were fragmented in the HCD trap with a normalised collision energy set to 30 . An underfill ratio at $1.0 \%$ with intensity threshold at $1.0 \times 10^{5}$ was used. Apex triggering was set to $3 s-10 s$ and dynamic exclusion was set to $30 \mathrm{~s}$. The sample was analyzed in three technical replicates.

2.4. Protein Identification and Bioinformatics. Data obtained by mass spectrometry were searched against a pig isoform database optimized from Uniprot using MaxQuant (version 1.5.0.22). The label-free algorithm was activated in MaxQuant. The peptide and protein false discovery rates were set to $1 \%$. A two-tailed, heteroscedastic $t$-test was used to calculate $p$ values.

Identified proteins were uploaded onto Perseus (version 1.5.0.9). Technical replicates were averaged by mean and proteins identified by less than two unique peptides were removed from the dataset. Perseus was used to search for proteins that were identified from peptides that were found to be part of a protein derived from the reversed part of the decoy database. These proteins were also removed from the dataset. All identified proteins were exported to Excel (Microsoft Office Standard 2007). Remaining keratins in the dataset were removed. Proteins with no assigned gene name in the identification were searched for on http://www.uniprot.com/ and gene names corresponding to the identified proteins were added.

A bioinformatic analysis was conducted using GeneCodis3 [18-20]. Gene names corresponding to the identified proteins were entered in GeneCodis3 to conduct Gene Ontology cellular component analysis. Homo sapiens was selected as organism to ensure that all gene names were recognized by GeneGodis3. $p$ values were obtained by

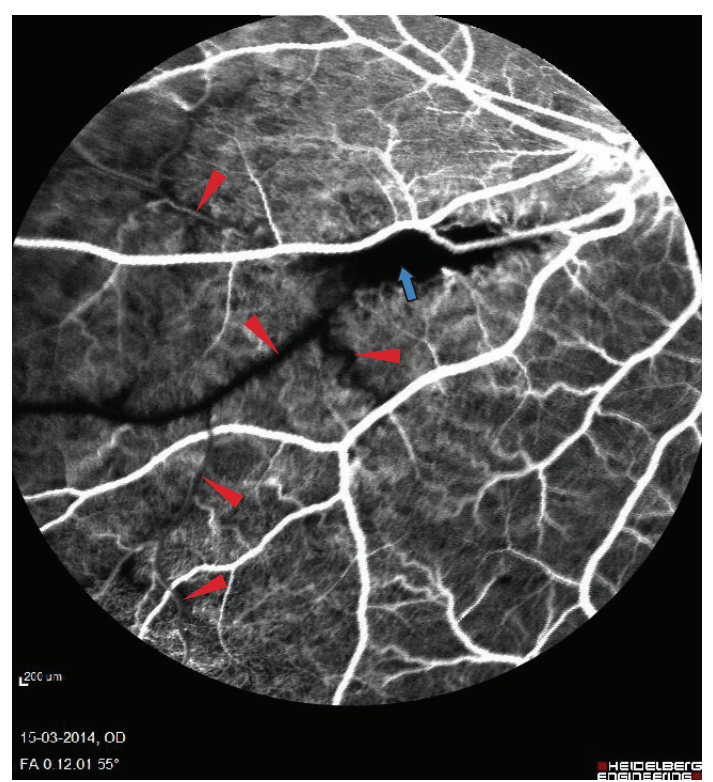

Figure 2: Delayed filling of occluded vein. The delayed filling is marked with red triangle. The site of occlusion is marked with blue arrow.

hypergeometric analysis corrected by a false discovery rate method.

\section{Results and Discussion}

Fluorescein angiography nine days after experimental BRVO showed delayed venous filling of the occluded vein (Figure 2). Drainage of venous blood by adjacent veins (Figure 3) was observed in the angiography after approximately 12-14 seconds and peripheral haemorrhages (Figure 4) appeared after 30-40 seconds. The vein remained occluded for the entire duration of the angiography that lasted close to 12 minutes. Ophthalmoscopic examination by day 15 showed the retinal vein to be entirely occluded. Peripheral haemorrhages had been absorbed and no retinal edema was observed.

After filtering by the criteria described above the proteomic analysis provided identification of 2686 proteins in the retina that was exposed to BRVO. Gene Ontology analysis showed that a large variety of proteins were identified in the retinal sample (Table 1).

3.1. Dye-Free Photothrombosis versus Photothrombosis with Administration of Rose Bengal. Donati et al. [16] used a Rose Bengal concentration of $10 \mathrm{mg} / \mathrm{kg}$ that was injected intravenously in minipigs of 10 to $12 \mathrm{~kg}$. BRVO was induced by applying argon green laser (wavelength $514 \mathrm{~nm}$ ) on a branch vein close to the optic nerve head using an energy intensity of $250 \mathrm{~mW}$, a pulse duration of 0.2 seconds, and a spot diameter of $500 \mu \mathrm{m}$ [16]. In our experiment without Rose Bengal the retinal vein remained unaffected when exposed to laser applications at $250 \mathrm{~mW}$ suggesting that the use of Rose 


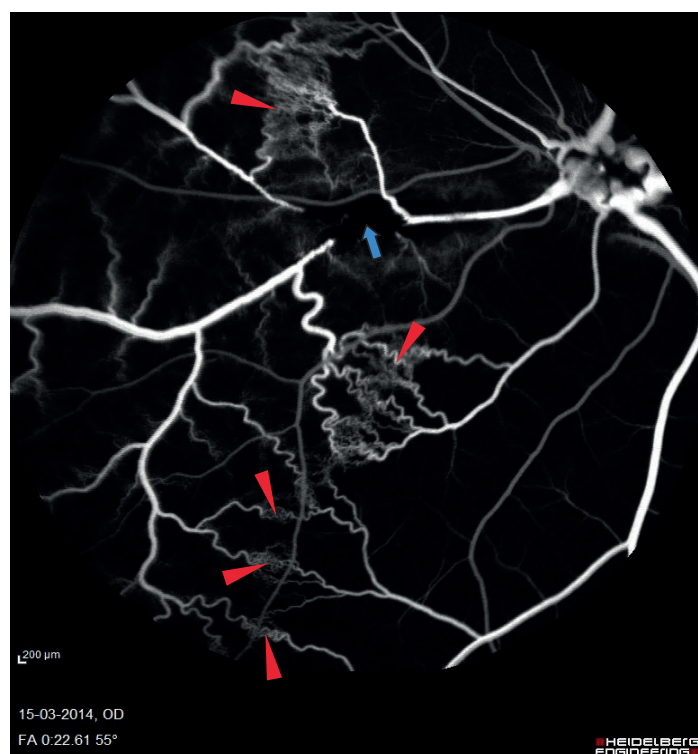

FIGURE 3: Shunting of blood for drainage by adjacent veins. Shunting of blood to adjacent veins is marked with red triangle. The site of occlusion is marked with blue arrow.

Bengal reduces the amount of energy that is needed for BRVO to occur.

In a study on porcine eyes by McAllister et al. [15] an intravenous injection of $10 \mathrm{mg} / \mathrm{kg}$ of Rose Bengal was injected via an ear vein and BRVO was induced with an argon green laser (wavelength $514 \mathrm{~nm}$ ) applied via a slit-lamp using the central position of a 3-mirror contact lens. With a spot size of $125 \mu \mathrm{m}$, power of $150 \mathrm{~mW}$, and duration of 1 second repeated laser burns (approximately 4-5) were applied directly to the inferior branch retinal vein until stagnation of the retinal blood flow was observed [15]. Though a duration of 1 second per laser application is a long exposure time, our experiment may indicate that a larger amount of laser applications is necessary to induce BRVO without Rose Bengal dye. Few laser applications may be an advantage in studies in which creation of the venous thrombus with minimal damage to the retinal vein is important.

Alternatively, BRVO can be induced in a porcine retina by establishing three sclerotomies in the porcine eye as in a three-port pars plana vitrectomy. By slowly lowering the diathermy probe toward the point of diathermia BRVO is induced by touching the vein with the diathermy probe for 6 seconds. No vitrectomy is performed [13]. However, this model requires access to surgical facilities and may affect the proteome of the vitreoretinal interface as the diathermy probe is in direct contact with the vitreous body and the retina.

3.2. Protein Modifications Induced by Rose Bengal. When exposed to visible light and oxygen, Rose Bengal produces singlet oxygen and reactive oxidative species, which can induce a number of protein modifications and interactions that may potentially affect the outcome of the proteomic study conducted [21]. Singlet oxygen $\left({ }^{1} \mathrm{O}_{2}\right)$ is known to oxidize the free amino acids Trp, Tyr, His, Cys, and Met

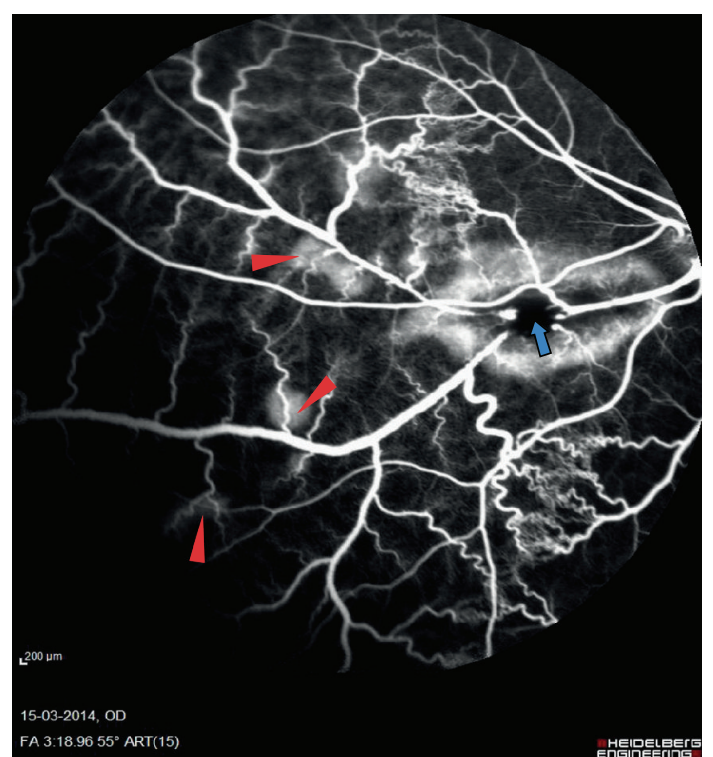

FIgURE 4: Peripheral haemorrhages. Peripheral haemorrhages appearing upstream to the occlusion are marked with red triangle. The site of occlusion is marked with blue arrow.

resulting in alterations to protein structure and function [2224]. These ${ }^{1} \mathrm{O}_{2}$-mediated changes include oxidation of side chains, dimerization, aggregation, unfolding, conformational changes, and alterations in cellular handling and turnover [24]. In a study by Rahmanto et al. the thioredoxin reductase and glutathione peroxidase as purified proteins and as cell lysates were exposed to Rose Bengal and visible light which resulted in photolysis induced reductions in the activity of the enzymes [22].

Menon et al. [25] demonstrated that irradiation of retinal pigment epithelium (RPE) cells with visible light in the presence of Rose Bengal dye induces RPE cell lysis in vitro [25]. Rose Bengal has been shown to bind to albumin [26] which makes the dye likely to be distributed to several retinal layers when the blood-retinal barrier is disrupted due to BRVO. Furthermore, Rose Bengal has been shown to induce secondary and tertiary structure alternations of bovine $\alpha$ crystallin in the presence of visible light [27].

3.3. Laser-Induced Effects on Protein Expression. Laser photocoagulation may potentially affect the retinal proteome. For example, in a proteome study by Quin et al. [28] laser photocoagulation was found to induce a downregulation of Wnt- 5 beta and calretinin in Dark Agouti rats with streptozotocin-induced diabetes mellitus.

To measure the proteome change caused by BRVO and avoid the measurement of protein changes caused by laser treatment it is essential to create an adequate control with an area of laser burns that is as identical as possible to the eye with BRVO. The laser exposure in the eye with BRVO should be sufficient for the BRVO to be induced, but unnecessary laser should be avoided. 
TABLE 1: Gene Ontology cellular compartment analysis.

\begin{tabular}{|c|c|c|c|c|}
\hline Annotations & Proteins & NGR & Hyp & Hyp $^{*}$ \\
\hline GO:0005737: cytoplasm & 1106 & 5302 & $1.8 \times 10^{-289}$ & $1.3 \times 10^{-286}$ \\
\hline GO:0005634: nucleus & 803 & 5441 & $1.2 \times 10^{-101}$ & $2.14 \times 10^{-99}$ \\
\hline GO:0005829: cytosol & 633 & 2146 & $4.4 \times 10^{-234}$ & $1.6 \times 10^{-231}$ \\
\hline GO:0016020: membrane & 551 & 4065 & $1.2 \times 10^{-52}$ & $9.2 \times 10^{-51}$ \\
\hline GO:0005886: plasma membrane & 472 & 3575 & $3.1 \times 10^{-41}$ & $1.4 \times 10^{-39}$ \\
\hline GO:0005739: mitochondrion & 453 & 1394 & $1.8 \times 10^{-181}$ & $4.3 \times 10^{-179}$ \\
\hline GO:0016021: integral to membrane & 405 & 4400 & $6.1 \times 10^{-8}$ & $5.2 \times 10^{-7}$ \\
\hline GO:0005730: nucleolus & 320 & 1461 & $6.2 \times 10^{-76}$ & $8.8 \times 10^{-74}$ \\
\hline GO:0005622: intracellular & 230 & 1962 & $8.8 \times 10^{-14}$ & $1.6 \times 10^{-12}$ \\
\hline GO:0005783: endoplasmic reticulum & 222 & 1000 & $2.8 \times 10^{-53}$ & $2.5 \times 10^{-51}$ \\
\hline GO:0005654: nucleoplasm & 215 & 891 & $4.3 \times 10^{-58}$ & $4.36 \times 10^{-56}$ \\
\hline GO:0005856: cytoskeleton & 187 & 846 & $1.4 \times 10^{-44}$ & $8.4 \times 10^{-43}$ \\
\hline GO:0005794: Golgi apparatus & 174 & 958 & $3.5 \times 10^{-30}$ & $1.2 \times 10^{-28}$ \\
\hline GO:0005576: extracellular region & 170 & 1913 & 0.003 & 0.008 \\
\hline GO:0005789: endoplasmic reticulum membrane & 140 & 642 & $7.3 \times 10^{-33}$ & $2.7 \times 10^{-31}$ \\
\hline GO:0005625: soluble fraction & 120 & 390 & $4.1 \times 10^{-44}$ & $2.2 \times 10^{-42}$ \\
\hline GO:0048471: perinuclear region of cytoplasm & 115 & 417 & $3.3 \times 10^{-37}$ & $1.4 \times 10^{-35}$ \\
\hline GO:0005743: mitochondrial inner membrane & 113 & 308 & $2.8 \times 10^{-50}$ & $2.0 \times 10^{-48}$ \\
\hline GO:0005759: mitochondrial matrix & 104 & 201 & $3.3 \times 10^{-64}$ & $3.9 \times 10^{-62}$ \\
\hline GO:0005624: membrane fraction & 98 & 523 & $2.0 \times 10^{-18}$ & $5.0 \times 10^{-42}$ \\
\hline GO:0005615: extracellular space & 96 & 784 & $2.5 \times 10^{-7}$ & $2.1 \times 10^{-6}$ \\
\hline GO:0005887: integral to plasma membrane & 94 & 1016 & 0.008 & 0.02 \\
\hline GO:0030054: cell junction & 85 & 475 & $5.9 \times 10^{-15}$ & $1.1 \times 10^{-13}$ \\
\hline GO:0005840: ribosome & 78 & 174 & $1.5 \times 10^{-42}$ & $7.1 \times 10^{-41}$ \\
\hline GO:0000139: Golgi membrane & 75 & 420 & $2.6 \times 10^{-13}$ & $4.4 \times 10^{-12}$ \\
\hline GO:0045202: synapse & 70 & 288 & $8.86 \times 10^{-20}$ & $2.4 \times 10^{-18}$ \\
\hline GO:0030529: ribonucleoprotein complex & 69 & 132 & $2.2 \times 10^{-43}$ & $1.1 \times 10^{-41}$ \\
\hline GO:0031410: cytoplasmic vesicle & 67 & 287 & $5.2 \times 10^{-18}$ & $1.3 \times 10^{-16}$ \\
\hline GO:0005792: microsome & 64 & 279 & $7.3 \times 10^{-17}$ & $1.6 \times 10^{-15}$ \\
\hline GO:0005813: centrosome & 63 & 323 & $3.7 \times 10^{-13}$ & $6.1 \times 10^{-12}$ \\
\hline GO:0042470: melanosome & 59 & 89 & $1.1 \times 10^{-45}$ & $7.0 \times 10^{-44}$ \\
\hline GO:0005874: microtubule & 59 & 262 & $2.8 \times 10^{-15}$ & $5.7 \times 10^{-14}$ \\
\hline GO:0009986: cell surface & 57 & 297 & $9.6 \times 10^{-12}$ & $1.3 \times 10^{-10}$ \\
\hline GO:0043231: intracellular membrane-bounded organelle & 56 & 269 & $4.7 \times 10^{-13}$ & $7.4 \times 10^{-12}$ \\
\hline GO:0015629: actin cytoskeleton & 54 & 180 & $5.16 \times 10^{-20}$ & $1.52 \times 10^{-18}$ \\
\hline GO:0043234: protein complex & 53 & 217 & $2.0 \times 10^{-15}$ & $4.0 \times 10^{-14}$ \\
\hline GO:0043025: neuronal cell body & 48 & 217 & $2.1 \times 10^{-12}$ & $3.2 \times 10^{-11}$ \\
\hline GO:0005694: chromosome & 48 & 265 & $3.0 \times 10^{-9}$ & $3.3 \times 10^{-8}$ \\
\hline GO:0071013: catalytic step 2 spliceosome & 47 & 79 & $1.8 \times 10^{-33}$ & $7.1 \times 10^{-32}$ \\
\hline GO:0016607: nuclear speck & 46 & 134 & $6.8 \times 10^{-20}$ & $1.9 \times 10^{-18}$ \\
\hline GO:0005768: endosome & 45 & 280 & $3.5 \times 10^{-7}$ & $2.6 \times 10^{-6}$ \\
\hline GO:0030424: axon & 43 & 173 & $4.6 \times 10^{-13}$ & $7.3 \times 10^{-12}$ \\
\hline GO:0030425: dendrite & 42 & 182 & $1.2 \times 10^{-11}$ & $1.5 \times 10^{-20}$ \\
\hline GO:0005764: lysosome & 40 & 179 & $1.0 \times 10^{-10}$ & $1.2 \times 10^{-9}$ \\
\hline GO:0019717: synaptosome & 39 & 111 & $1.7 \times 10^{-17}$ & $4.1 \times 10^{-16}$ \\
\hline GO:0042995: cell projection & 39 & 158 & $6.9 \times 10^{-12}$ & $9.8 \times 10^{-11}$ \\
\hline GO:0022625: cytosolic large ribosomal subunit & 38 & 52 & $1.9 \times 10^{-32}$ & $6.9 \times 10^{-31}$ \\
\hline GO:0005741: mitochondrial outer membrane & 37 & 102 & $3.4 \times 10^{-17}$ & $7.8 \times 10^{-16}$ \\
\hline GO:0031012: extracellular matrix & 37 & 146 & $1.0 \times 10^{-11}$ & $1.3 \times 10^{-11}$ \\
\hline GO:0000502: proteasome complex & 36 & 60 & $3.8 \times 10^{-26}$ & $1.2 \times 10^{-24}$ \\
\hline
\end{tabular}


TABle 1: Continued.

\begin{tabular}{|c|c|c|c|c|}
\hline Annotations & Proteins & NGR & Hyp & Hyp $^{*}$ \\
\hline GO:0016323: basolateral plasma membrane & 35 & 129 & $4.4 \times 10^{-12}$ & $6.4 \times 10^{-11}$ \\
\hline GO:0005681: spliceosomal complex & 34 & 75 & $1.6 \times 10^{-19}$ & $4.1 \times 10^{-18}$ \\
\hline GO:0016324: apical plasma membrane & 32 & 196 & $1.2 \times 10^{-5}$ & $6.4 \times 10^{-5}$ \\
\hline GO:0005938: cell cortex & 31 & 106 & $8.7 \times 10^{-12}$ & $1.2 \times 10^{-10}$ \\
\hline GO:0005635: nuclear envelope & 31 & 107 & $1.1 \times 10^{-11}$ & $1.5 \times 10^{-10}$ \\
\hline GO:0031965: nuclear membrane & 30 & 133 & $1.7 \times 10^{-8}$ & $1.6 \times 10^{-7}$ \\
\hline GO:0005911: cell-cell junction & 29 & 93 & $6.9 \times 10^{-12}$ & $9.6 \times 10^{-11}$ \\
\hline GO:0005788: endoplasmic reticulum lumen & 29 & 106 & $2.4 \times 10^{-10}$ & $2.8 \times 10^{-9}$ \\
\hline GO:0005925: focal adhesion & 28 & 116 & $1.0 \times 10^{-8}$ & $1.1 \times 10^{-7}$ \\
\hline GO:0030496: midbody & 27 & 73 & $3.6 \times 10^{-13}$ & $6.0 \times 10^{-12}$ \\
\hline GO:0005769: early endosome & 27 & 122 & $1.3 \times 10^{-7}$ & $1.1 \times 10^{-6}$ \\
\hline GO:0022627: cytosolic small ribosomal subunit & 26 & 36 & $2.2 \times 10^{-22}$ & $6.8 \times 10^{-21}$ \\
\hline GO:0015630: microtubule cytoskeleton & 26 & 97 & $3.2 \times 10^{-9}$ & $3.4 \times 10^{-8}$ \\
\hline GO:0045211: postsynaptic membrane & 26 & 170 & 0.0002 & 0.0009 \\
\hline GO:0005643: nuclear pore & 25 & 58 & $4.1 \times 10^{-14}$ & $7.7 \times 10^{-13}$ \\
\hline GO:0045121: membrane raft & 25 & 128 & $4.3 \times 10^{-6}$ & $2.5 \times 10^{-5}$ \\
\hline GO:0009897: external side of plasma membrane & 25 & 155 & 0.0001 & 0.0005 \\
\hline GO:0008021: synaptic vesicle & 24 & 70 & $4.7 \times 10^{-11}$ & $5.7 \times 10^{-10}$ \\
\hline GO:0005777: peroxisome & 24 & 105 & $3.4 \times 10^{-7}$ & $2.6 \times 10^{-11}$ \\
\hline GO:0014069: postsynaptic density & 23 & 107 & $1.9 \times 10^{-6}$ & $1.2 \times 10^{-5}$ \\
\hline GO:0005793: endoplasmic reticulum-Golgi intermediate compartment & 22 & 46 & $9.8 \times 10^{-14}$ & $1.7 \times 10^{-12}$ \\
\hline GO:0005819: spindle & 22 & 113 & $1.7 \times 10^{-5}$ & $8.9 \times 10^{-5}$ \\
\hline GO:0005765: lysosomal membrane & 22 & 123 & $6.4 \times 10^{-5}$ & 0.0003 \\
\hline GO:0010008: endosome membrane & 22 & 145 & 0.0007 & 0.003 \\
\hline GO:0001725: stress fiber & 21 & 45 & $6.6 \times 10^{-13}$ & $1.0 \times 10^{-11}$ \\
\hline GO:0030426: growth cone & 21 & 72 & $2.0 \times 10^{-8}$ & $1.9 \times 10^{-7}$ \\
\hline GO:0043005: neuron projection & 21 & 81 & $1.9 \times 10^{-7}$ & $1.6 \times 10^{-6}$ \\
\hline GO:0005905: coated pit & 20 & 46 & $1.2 \times 10^{-11}$ & $1.5 \times 10^{-10}$ \\
\hline GO:0031901: early endosome membrane & 20 & 63 & $8.7 \times 10^{-7}$ & $9.07 \times 10^{-8}$ \\
\hline GO:0001726: ruffle & 20 & 72 & $1.1 \times 10^{-7}$ & $9.2 \times 10^{-7}$ \\
\hline GO:0030659: cytoplasmic vesicle membrane & 20 & 83 & $1.3 \times 10^{-6}$ & $8.9 \times 10^{-6}$ \\
\hline GO:0030027: lamellipodium & 20 & 101 & $3.0 \times 10^{-5}$ & 0.0002 \\
\hline
\end{tabular}

Cellular compartments identified by at least 20 proteins are included.

Proteins: number of proteins identified in cellular compartment.

NGR: number of annotated proteins in the reference list.

Hyp: hypergeometric $p$ value.

Hyp $^{*}$ : corrected hypergeometric $p$ value.

\section{Conclusion}

In this paper we describe a novel optimized method for proteomic analysis of retinal tissue exposed to experimental BRVO by combining a dye-free photocoagulation based porcine model with liquid chromatography tandem mass spectrometry. Dye-free photocoagulation resulted in successful induction of BRVO. The following proteomic analysis of the retinal tissue resulted in identification of 2686 proteins of various cellular compartments. For proteomic analysis of retinal tissue BRVO is best conducted without the use of Rose Bengal to avoid the multiple protein modifications that are induced when the dye is irradiated. However, induction of BRVO without Rose Bengal dye may require a larger number of laser applications and a higher level of laser energy.
Therefore, creation of an adequate control with an area of laser burns that is similar to the eye with BRVO is essential to avoid measuring laser-induced proteome changes.

\section{Conflict of Interests}

All authors declare that there is no conflict of interests regarding the publication of this paper.

\section{Acknowledgments}

This work was supported by the Obel Family Foundation, the Herta Christensen Foundation, the Research Foundation of the North Denmark Region, the Bagger-Sørensen 
Foundation, and the Svend Andersen Foundation. The authors would like to thank Clinical Photographer Anita Irene Kristensen and Ophthalmic Nurse Bente Ditmann Pedersen from Department of Ophthalmology, Aalborg University Hospital, for their help with fundus photography and fluorescein angiography.

\section{References}

[1] S. L. Rogers, R. L. McIntosh, L. Lim et al., "Natural history of branch retinal vein occlusion: an evidence-based systematic review," Ophthalmology, vol. 117, no. 6, pp. 1094.e5-1101.e5, 2010.

[2] M. Bertelsen, A. Linneberg, T. Rosenberg et al., "Comorbidity in patients with branch retinal vein occlusion: case-control study," British Medical Journal, vol. 345, no. 7887, Article ID e7885, 2012.

[3] J. Zhao, S. M. Sastry, R. D. Sperduto et al., "Arteriovenous crossing patterns in branch retinal vein occlusion," Ophthalmology, vol. 100, no. 3, pp. 423-428, 1993.

[4] J. Rehak and M. Rehak, "Branch retinal vein occlusion: Pathogenesis, visual prognosis, and treatment modalities," Current Eye Research, vol. 33, no. 2, pp. 111-131, 2008.

[5] H. Noma, T. Mimura, and S. Eguchi, "Association of inflammatory factors with macular edema in branch retinal vein occlusion," JAMA Ophthalmology, vol. 131, no. 2, pp. 160-165, 2013.

[6] N. Mandal, S. Heegaard, J. U. Prause, B. Honoré, and H. Vorum, "Ocular proteomics with emphasis on two-dimensional gel electrophoresis and mass spectrometry," Biological Procedures Online, vol. 12, no. 1, pp. 56-88, 2010.

[7] B. Honoré and H. Vorum, "Proteomic analysis as a means to approach limbal stem cell biology in a search for stem cell markers," Proteomics-Clinical Applications, vol. 8, no. 3-4, pp. 178-184, 2014.

[8] N. Mandal, M. Kofod, H. Vorum et al., "Proteomic analysis of human vitreous associated with idiopathic epiretinal membrane," Acta Ophthalmologica, vol. 91, no. 4, pp. e333-e334, 2013.

[9] A. Decanini, P. R. Karunadharma, C. L. Nordgaard, X. Feng, T. W. Olsen, and D. A. Ferrington, "Human retinal pigment epithelium proteome changes in early diabetes," Diabetologia, vol. 51, no. 6, pp. 1051-1061, 2008.

[10] L. J. Cehofski, N. Mandal, B. Honoré, and H. Vorum, "Analytical platforms in vitreoretinal proteomics," Bioanalysis, vol. 6, no. 22, pp. 3051-3066, 2014.

[11] A. M. de Almeida and E. Bendixen, "Pig proteomics: a review of a species in the crossroad between biomedical and food sciences," Journal of Proteomics, vol. 75, no. 14, pp. 4296-4314, 2012.

[12] G. P. Lewis, D. G. Charteris, C. S. Sethi, and S. K. Fisher, "Animal models of retinal detachment and reattachment: identifying cellular events that may affect visual recovery," Eye, vol. 16, no. 4, pp. 375-387, 2002.

[13] R. Ejstrup, E. Scherfig, and M. la Cour, "Electrophysiological consequences of experimental branch retinal vein occlusion in pigs and the effect of dorzolamide," Investigative Ophthalmology \& Visual Science, vol. 52, no. 2, pp. 952-958, 2011.

[14] I. L. McAllister, S. Vijayasekaran, and D.-Y. Yu, "Intravitreal tenecteplase (Metalyse) for acute management of retinal vein occlusions," Investigative Ophthalmology and Visual Science, vol. 54, no. 7, pp. 4910-4918, 2013.

[15] I. L. McAllister, S. Vijayasekaran, S. D. Chen, and D.-Y. Yu, "Effect of triamcinolone acetonide on vascular endothelial growth factor and occludin levels in branch retinal vein occlusion," American Journal of Ophthalmology, vol. 147, no. 5, pp. 838.e2-846.e2, 2009.

[16] G. Donati, C. J. Pournaras, G.-P. Pizzolato, and M. Tsacopoulos, "Decreased nitric oxide production accounts for secondary arteriolar constriction after retinal branch vein occlusion," Investigative Ophthalmology \& Visual Science, vol. 38, no. 7, pp. 1450-1457, 1997.

[17] J. Larsson, J. Carlson, and S. B. Olsson, "Ultrasound enhanced thrombolysis in experimental retinal vein occlusion in the rabbit," British Journal of Ophthalmology, vol. 82, no. 12, pp. 1438-1440, 1998.

[18] P. Carmona-Saez, M. Chagoyen, F. Tirado, J. M. Carazo, and A. Pascual-Montano, "GENECODIS: a web-based tool for finding significant concurrent annotations in gene lists," Genome Biology, vol. 8, no. 1, article R3, 2007.

[19] R. Nogales-Cadenas, P. Carmona-Saez, M. Vazquez et al., "GeneCodis: interpreting gene lists through enrichment analysis and integration of diverse biological information," Nucleic Acids Research, vol. 37, supplement 2, pp. W317-W322, 2009.

[20] D. Tabas-Madrid, R. Nogales-Cadenas, and A. PascualMontano, "GeneCodis3: a non-redundant and modular enrichment analysis tool for functional genomics," Nucleic Acids Research, vol. 40, no. 1, pp. W478-W483, 2012.

[21] Y. C. Lee, C. K. Park, M. S. Kim, and J. H. Kim, “In vitro study for staining and toxicity of rose bengal on cultured bovine corneal endothelial cells," Cornea, vol. 15, no. 4, pp. 376-385, 1996.

[22] A. S. Rahmanto, D. I. Pattison, and M. J. Davies, "Photooxidation-induced inactivation of the selenium-containing protective enzymes thioredoxin reductase and glutathione peroxidase," Free Radical Biology and Medicine, vol. 53, no. 6, pp. 13081316, 2012.

[23] M. J. Davies, "Reactive species formed on proteins exposed to singlet oxygen," Photochemical \& Photobiological Sciences, vol. 3, no. 1, pp. 17-25, 2004.

[24] A. Wright, W. A. Bubb, C. L. Hawkins, and M. J. Davies, "Singlet oxygen-mediated protein oxidation: evidence for the formation of reactive side chain peroxides on tyrosine residues," Photochemistry and Photobiology, vol. 76, no. 1, pp. 35-46, 2002.

[25] I. A. Menon, P. K. Basu, S. D. Persad, S. Rosatone, and J. D. Wiltshire, "A study on the sequence of phototoxic effects of rose bengal using retinal pigment epithelial cells in vitro," Experimental Eye Research, vol. 49, no. 1, pp. 67-73, 1989.

[26] S. C. G. Tseng and S.-H. Zhang, "Interaction between rose bengal and different protein components," Cornea, vol. 14, no. 4, pp. 427-435, 1995.

[27] T. Youssef, M. Kassem, T. Abdella, M. A. Harith, and F. Lenci, "Photosensitized effects of rose bengal on structure and function of lens protein 'alpha-crystallin"' Photochemistry and Photobiology, vol. 85, no. 6, pp. 1306-1313, 2009.

[28] G. J. Quin, B. Lyons, A. C. Len, M. C. Madigan, and M. C. Gillies, "Proteome changes induced by laser in diabetic retinopathy," Clinical \& Experimental Ophthalmology, vol. 43, no. 2, pp. 180187, 2015. 


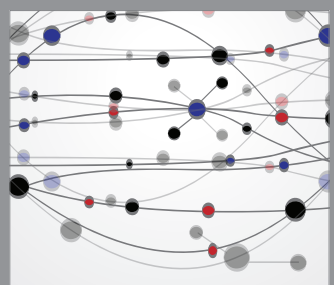

The Scientific World Journal
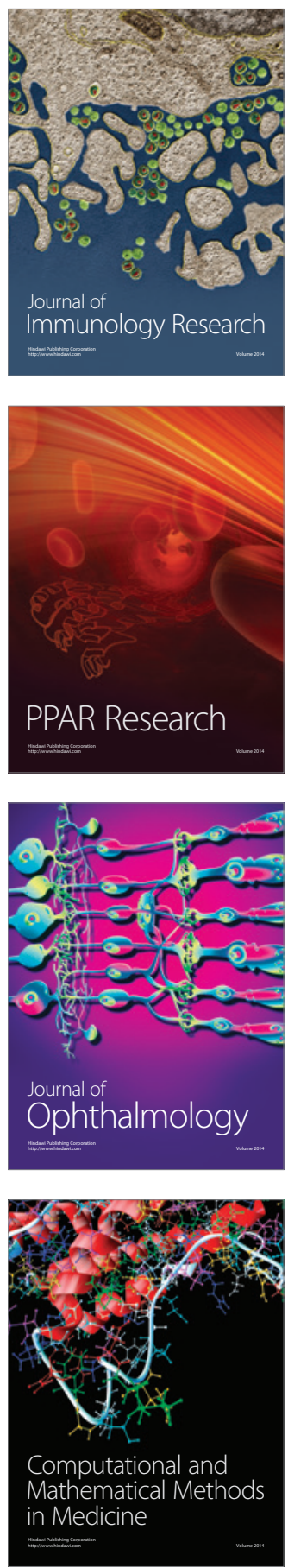

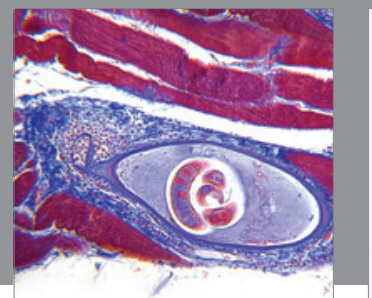

Gastroenterology

Research and Practice
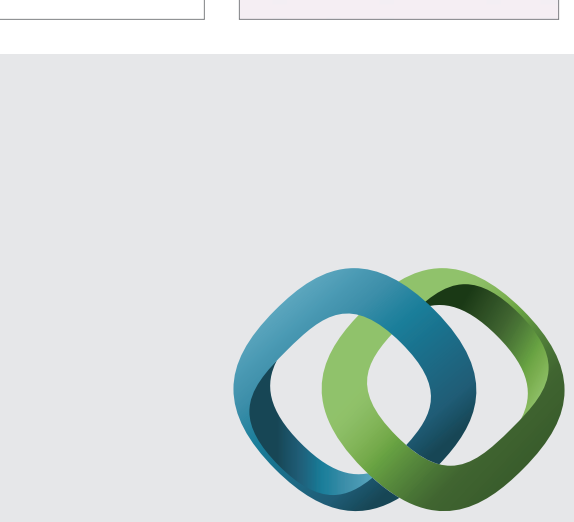

\section{Hindawi}

Submit your manuscripts at

http://www.hindawi.com
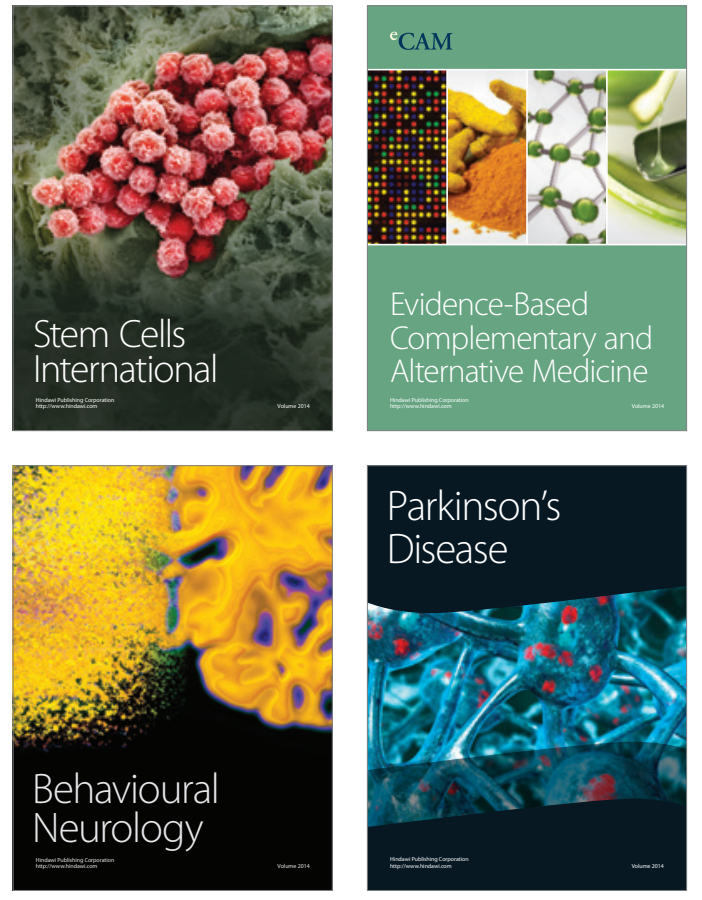
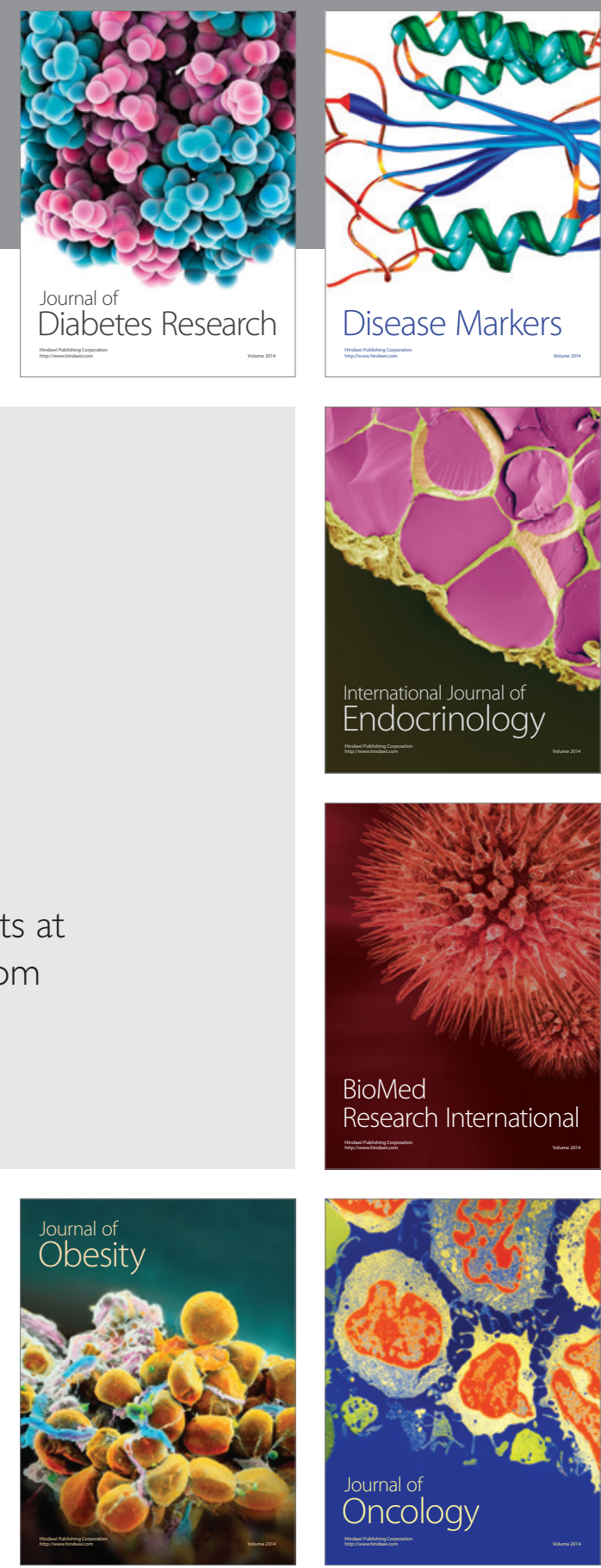

Disease Markers
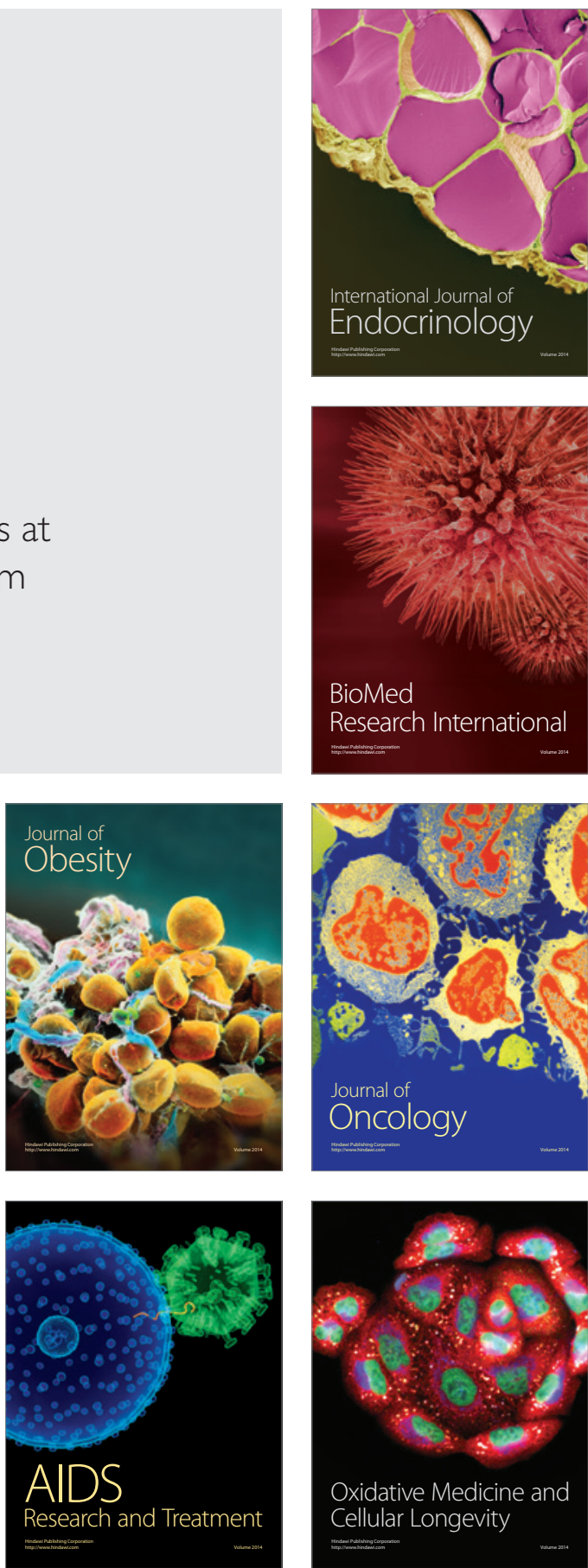